\title{
A parametrically excited vibration energy harvester
}

\author{
Yu Jia, Jize Yan, Kenichi Soga and Ashwin A. Seshia* \\ Department of Engineering, University of Cambridge, Cambridge CB2 1PZ, UK
}

Draft: March 22, 2013

\begin{abstract}
In the arena of vibration energy harvesting, the key technical challenges continue to be low power density and narrow operational frequency bandwidth. While the convention has relied upon the activation of the fundamental mode of resonance through direct excitation, this paper explores a new paradigm through the employment of parametric resonance. Unlike the former, oscillatory amplitude growth is not limited due to linear damping. Therefore, the power output can potentially build up to higher levels. Additionally, it is the onset of nonlinearity that eventually limits parametric resonance; hence, this approach can also potentially broaden the operating frequency range. Theoretical prediction and numerical modelling have suggested an order higher in oscillatory amplitude growth. Experimentally, an initial macrosized electromagnetic prototype (practical volume $\sim 1,800 \mathrm{~cm}^{3}$ ), when parametrically driven, has demonstrated around 50\% increase in half power band and an order of magnitude higher peak power density normalised against input acceleration squared $\left(293 \mu \mathrm{Wcm}^{-3} \mathrm{~m}^{-2} \mathrm{~s}^{4}\right.$ with $171.5 \mathrm{~mW}$ at $0.57 \mathrm{~ms}^{-2}$ ) in contrast to the same prototype directly driven at fundamental resonance $(36.5$ $\mu \mathrm{Wcm}^{-3} \mathrm{~m}^{-2} \mathrm{~s}^{4}$ with $27.75 \mathrm{~mW}$ at $0.65 \mathrm{~ms}^{-2}$ ). This figure suggests promising potentials while comparing with current state-of-the-art macro-sized counterparts, such as Perpetuum's PMG-17 $\left(119 \mu \mathbf{W c m}^{-3} \mathbf{m}^{-2} \mathbf{s}^{4}\right)$.
\end{abstract}

Keywords: parametric resonance, electromagnetic, vibration, energy harvesting

\section{Introduction}

In the past decade, energy harvesting has witnessed a rapid increase of interest from both academia and industry [1]. In contrast to the top down process of conventional power generation, the decentralised and self-sustaining nature of energy harvesting provides a convenient onboard complement to batteries for prolonged lifetime of remote and wireless devices. For an overview of developments in this field, readers can refer to review articles such as those by Beeby et al. (2006) [2] and Mitcheson el al. (2008) [3], as well as a textbook published in 2009 [1].

Solar power has already emerged as a relatively mature technology for decentralised power generation; however, it is not suitable for enclosed or embedded applications where luminosity is scarce [4]. On the other hand, ambient kinetic vibration is observed in many applications such as railways, bridges, industrial machinery and human body [3].

Most reported vibration harvesters rely on the activation of the fundamental mode of resonance through direct excitation of a second order mass-spring-damper system [1], where the driving force is typically applied parallel to the direction of the oscillatory displacement. The fundamental mode of resonance is attained when the excitation frequency matches the resonant frequency of the system. This type of resonance, achieved through direct excitation, is defined as 'ordinary resonance' within the context of this paper for the purpose of clarity.

Two major persisting technical challenges of this emerging technology are the small power density and narrow operational frequency bandwidth. Due to the random and continuously varying nature of real world vibrational sources, such as the example shown in figure 1, an ideal harvester should be able to function over a wide range of frequencies. However, designing a system with a flatter resonant response through the tuning of damping, compromises the peak power achievable. Therefore, the ideal objective is to maximise both the peak and the frequency bandwidth.

In an attempt to resolve this dilemma, this paper departs from the convention (ordinary resonance) and investigates the employment of parametric resonance as a means of mechanical amplification while

\footnotetext{
${ }^{*}$ Corresponding author. Tel.: +44(0)1223 760333; fax: +44(0)1223 760309. E-mail address: aas41@cam.ac.uk
} 


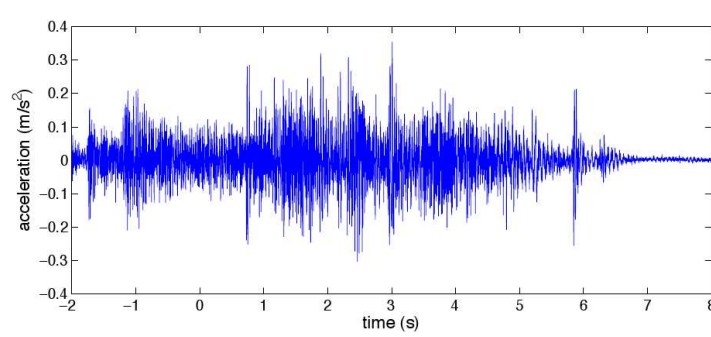

(a) Time domain of recorded data

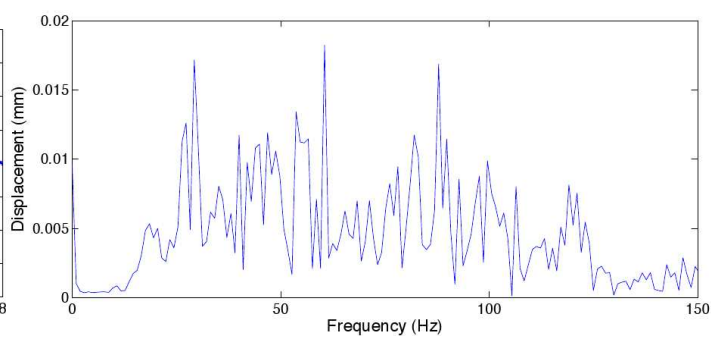

(b) Frequency domain calculated through Fast Fourier Transform

Figure 1: A typical sample of real vibration measured from a railway bridge (1 inch from the rail tracks). Random vibration can be observed with several significant peaks covering a broad frequency range below $100 \mathrm{~Hz}$.

exploiting its nonlinear resonant characteristics at high amplitudes to widen the frequency band. This particular resonant phenomenon is induced when an external excitation results in a periodic modulation of an internal system parameter. In contrast to ordinary resonance, the driving force is usually perpendicular to the direction of the oscillatory displacement.

To date, only one previous study (to the best knowledge of the authors) of utilising parametric resonance for vibration energy harvesting has been investigated [5] and significant performance enhancements have yet to be reported. One of the main limiting factors of this approach is the requirement for the excitation amplitude to exceed a certain initiation threshold prior to accessing the parametric resonant regime. A novel design and working mechanism are investigated in this study in order to reduce the shortcomings of a parametrically excited vibration energy harvester (PEVEH) for practical realisation.

\section{Theory and motivation}

One of the first documented reports of parametric resonance was by Michael Faraday in 1831 [6, 7] upon observing that a vertically oscillating cylinder on the surface of a fluid had half the frequency of the excitation. An early experimental investigation was carried out by Lord Rayleigh in 1883 [7, 8], where a taut string was attached to a tuning fork; when the tuning fork vibrated with a frequency $f$, the string experienced lateral vibration with half $f$. Since then, the fundamental principles of this phenomenon have been comprehensively covered in various texts $[7,9,10]$.

Parametric resonance is distinct from most vibrational resonances due to a time-dependent modulation in at least one of its system parameters [9]. There are two classifications: heteroparametric resonance (which is simply referred to as parametric resonance in modern academia) and autoparametric resonance [7]. Heteroparametric excitation is induced by the periodic modulation of certain system parameters in response to an external force. Meanwhile, autoparametric resonance arises from certain integer ratio relationships among the various natural frequencies of a multiple degree-of-freedom system, resulting in one oscillating component of the system introducing a periodic modulation of the system parameter on a second oscillator. Mathematically, both types of parametric resonance can be described by the same generic equation [11].

The motivation for this study is inspired by a pivotal advantage, as outlined in Table 1, which can potentially enable a significant leap forward in performance over the current paradigm of vibration energy harvesting. Unlike directly excited ordinary resonance, oscillatory amplitude growth due to parametric resonance does not saturate by linear damping and can only be limited by either physical limits or the onset of non-linearity at high amplitudes. This rise of nonlinearity that is almost always associated with parametric resonance can further aid the widening of frequency band, thereby fulfilling the two dilemmatic objectives simultaneously as summarised below.

- Using parametric resonance as a means of mechanical amplification to maximise the power peak

- Using its nonlinear resonant peak to broaden the operational frequency bandwidth

Unlike systems under direct excitation, the homogeneous parts of the equation of motion of a parametrically excited system contain functions of time as shown in equation 1.

$$
\ddot{x}+p(t) \dot{x}+q(t) x=f(t)
$$


Table 1: Motivation for employing parametric resonance over ordinary resonance. Energy invested $E_{\text {in }}$ by the former is directly proportional to energy dissipation by linear damping $E_{l o s t}$ while the latter is proportional to $\sqrt{E_{\text {lost }}}$. Therefore, theory predicts an order higher in oscillatory amplitude growth over ordinary resonance [12].

\begin{tabular}{c||c|c|c}
\hline \hline \multicolumn{1}{c||}{$\begin{array}{c}\text { Energy input } \\
E_{\text {in }}\end{array}$} & \multicolumn{3}{c}{ Directly proportional to, $\propto$} \\
\cline { 2 - 4 } & Amplitude growth & Energy stored & Energy dissipated by linear damping \\
\hline \hline Ordinary & $A$ & $\sqrt{E_{\text {stored }}}$ & $\sqrt{E_{\text {lost }}}$ \\
\hline Parametric & $A^{2}$ & $E_{\text {stored }}$ & $E_{\text {lost }}$ \\
\hline \hline
\end{tabular}

where, $x$ is a generic displacement parameter, $p$ is a generic damping parameter, $q$ is a generic restoring force parameter, $f$ is a generic external forcing and $t$ is the time domain. The parametric modulation of $p(t)$ and $q(t)$ can act as excitation terms as well. For the particular case of $p(t)=0$ and $f(t)=0$, the following generic undamped Mathieu eqiation can be derived [11].

$$
\ddot{x}+c \dot{x}+(\delta+2 \varepsilon \cos (2 t)) x=0
$$

where, $c$ is the damping coefficient, $\delta$ and $\varepsilon$ are generic parameters related to the square of natural frequency and amplitude of the parametric excitation respectively. The values of $\delta$ and $\varepsilon$ determine the stability of the system [7] as illustrated in the stability chart diagram in figure 2 . When displacement $x$ has unbounded solutions, an exponential build up of oscillatory amplitude can be achieved. This amplitude growth can theoretically approach infinity in a hypothetical purely linear setting and is represented by the unstable region (shaded) in the stability chart. The first order or the principal parametric resonance, which is usually observed at double the natural frequency of a periodically excited system (as presented in Section III), exhibits the largest unstable region. Although higher orders of parametric resonance is theoretically possible, it is harder to attain as the stable region starts to become more predominant.
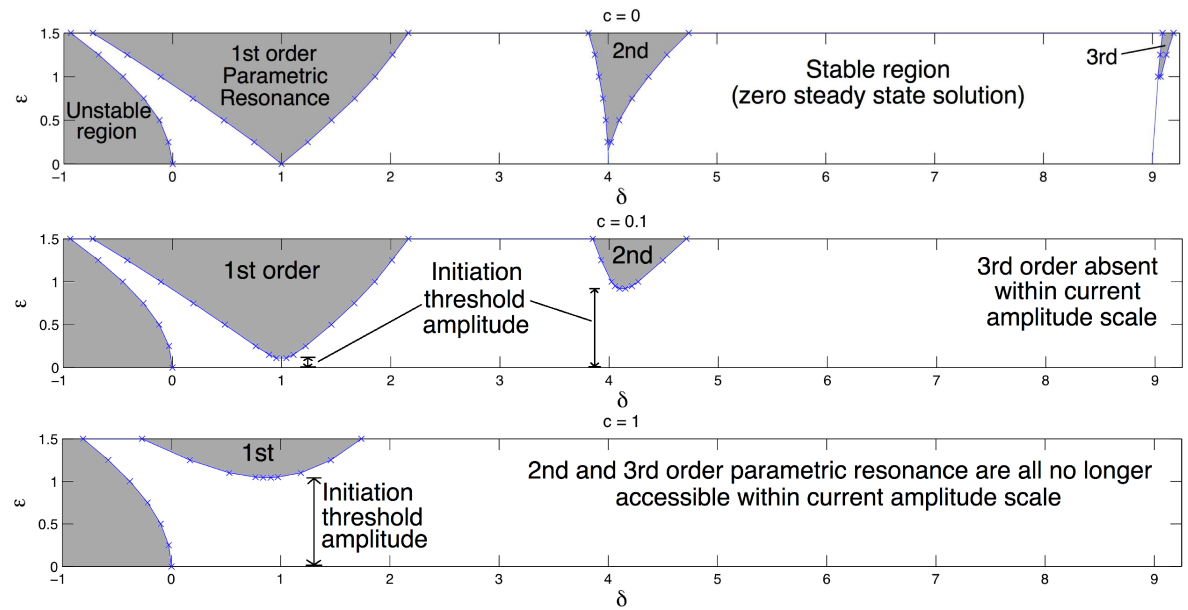

Figure 2: Stability chart in the $\delta$ - $\varepsilon$ parametric plane of Mathieu equation with varying damping term ' $c$ '. Stable regions are unshaded and unstable regions are shaded. The unstable regions signify the achievement of parametric resonance. A damping dependent initiation threshold amplitude is present. Therefore, at higher damping, larger amplitudes are required to activate parametric resonance.

Despite the promising potentials, not all system configurations will enjoy the advantages of larger amplitudes from parametric resonance at attainable excitation levels. One of the main hindrance factors is the presence of a damping dependent initiation threshold (see figure 2), which the excitation amplitude must attain. If the excitation is below this threshold, the system would be trapped within a stable equilibrium as experienced and reported by Daqaq et al. [5]. In addition to the frequency and amplitude conditions, an initial non-zero displacement is also required to 'push' the system out of stable equilibrium.

Parametric resonance has been widely observed to attain significant amplitudes and its traditional study has involved applications to inhibit its onset or limit its growth such as the prevention of mechanical failure like aircraft wings [10]. This enables the mechanism to potentially act as a mechanical amplifier for maximising the energy conversion efficiency of a given mechanical-to-electrical transducer and drastically improve its power density. 
Amplifiers using this phenomenon have already been explored in sensing applications such as MEMS gyroscopes [13, 14]. These designs typically rely on a drive actuator acting perpendicularly to the sensing mode in order to introduce a time varying coefficient in the equation of motion. Since drive actuators drain extra power, these design approaches are not viable for energy harvesting applications. The objective in the context of energy harvesting, therefore, is to derive a mechanical design that can passively induce parametric excitation while minimising the effect of electrical damping on the parametric resonator.

\section{Design and analytical model}

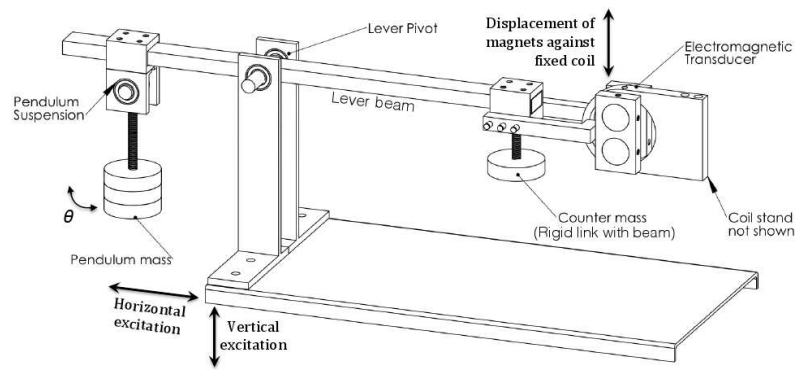

Figure 3: Design schematic of a Parametrically Excited Vibration Energy Harvester (PEVEH) prototype. Horizontal driving force yields direct excitation and vertical driving force yields parametric excitation.

The design schematic in figure 3 presents a macro scale Parametrically Excited Vibration Energy Harvester (PEVEH) prototype. Parametric excitation can be observed in a variety of systems depending on the precise excitation criteria. The pendulum suspended on the left-hand-side of this lever beam is one such system, which can be directly and/or parametrically driven as illustrated in figure 4. Propagation of vibration from the anchored base drives the pendulum with angular displacement $\theta(t)$. The lever mechanism enables further mechanical amplification on the transducer side with vertical displacement $y(t)$ assuming small arc angle.

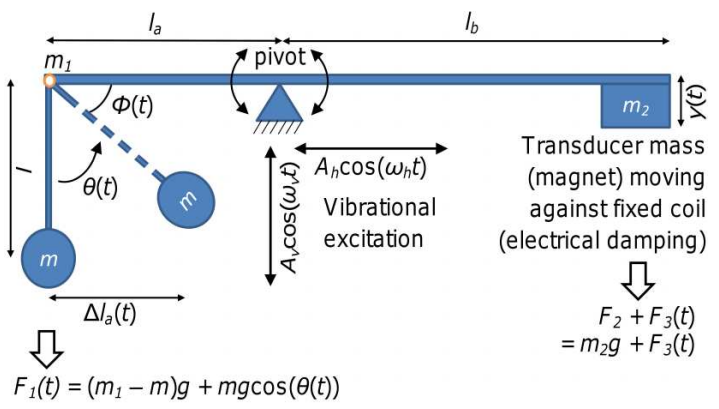

Figure 4: Working mechanism of the PEVEH system. Horizontally driving $\left(A_{h} \cos \omega_{h} t\right)$ the pendulum equates to direct excitation, which allows the activation of ordinary resonance when $\omega_{h}$ equals the pendulum's natural frequency $\omega_{0}$. On the other hand, vertically driving the pendulum $\left(A_{v} \cos \omega_{v} t\right)$ is a form of parametric excitation and can activate parametric resonance when $\omega_{v} \approx 2 \omega_{0}$. Displacement induced by pendulum motion is further mechanically amplified on the transducer side by the lever.

The principal damping (transducer's electrical damping) does not directly act on the pendulum. Therefore, the initiation amplitude threshold required to activate parametric resonance is lower in contrast to a design where the pendulum mass is primarily damped. This design is partially inspired from a two stage mechanical oscillator [15], typically implemented as a rural water pump.

Horizontally driving a pendulum at its suspension induces a direct excitation governed by equation 3 .

$$
\ddot{\theta}+c \dot{\theta}+\omega_{0}^{2} \sin \theta=\omega_{h}^{2} \frac{A_{h}}{l} \cos \left(\omega_{h} t\right)
$$

where, $\theta$ is the angular displacement of the pendulum, $\omega_{0}$ is the angular natural frequency of the pendulum, $\omega_{h}$ is the horizontal excitation angular frequency, $A_{h}$ is the horizontal excitation displacement amplitude, $c$ is the pendulum damping coefficient, $l$ is the pendulum arm length and $t$ is the time domain. 
With a vertical driving force, equation 4 governs the system's motion. The presence of a time varying coefficient implies that this is a damped Mathieu equation and parametric excitation can be initiated.

$$
\ddot{\theta}+c \dot{\theta}+\left(\omega_{0}^{2}+\omega_{v}^{2} \frac{A_{v}}{l} \cos \left(\omega_{v} t\right)\right) \sin \theta=0
$$

where, $\omega_{v}$ is the vertical excitation angular frequency and $A_{v}$ is the vertical excitation displacement amplitude. Equation 5 becomes the governing equation when both horizontal (direct) and vertical (parametric) excitations are present.

$$
\ddot{\theta}+c \dot{\theta}+\left(\omega_{0}^{2}+\omega_{v}^{2} \frac{A_{v}}{l} \cos \left(\omega_{v} t\right)\right) \sin \theta=\omega_{h}^{2} \frac{A_{h}}{l} \sin \left(\omega_{h} t\right)
$$

Ordinary resonance in equations 3 and 5 can be attained when $\omega_{h}=\omega_{0}$. Parametric resonance in equations 4 and 5 can be achieved when $\omega_{v}=2 \omega_{0} / n$; where $n$ is the order number. Principal parametric resonance is observed when $n=1$.

The core mechanism of the prototype, as shown in figure 4, involves the propagation of vibrational excitation along the system to drive the pendulum at its suspension. When angular displacement $\theta(t)$ is non-zero, the lever beam (acting as an additional mechanical amplifier) is unbalanced and drives the transducer in the vertical direction. $F_{1}(t)$ is the force exerted by the weight of pendulum, $F_{2}$ is the weight of the transducer side and $F_{3}(t)$ is the force acting on the lever beam due to the electrical damping of the transducer.

The equilibrium equations describing the lever beam balanced at rest $(t=0$ and $\theta=0)$ is given by equation 6 .

$$
\begin{array}{r}
F_{1}(t) l_{a}(t)=F_{2} l_{b} \\
\text { where, } F_{1}(t)=\left(m_{1}-m\right) g+m g \cos (\theta(t)) \\
\text { and, } F_{1}(0)=\left(m_{1}-m\right) g+m g \cos (0)=m_{1} g \\
\text { also, } F_{2}=m_{2} g \\
\text { therefore, } m_{1} g l_{a}(0)=m_{2} g l_{b}
\end{array}
$$

where, $m$ is the pendulum mass, $m_{1}$ is the total mass of the pendulum side, $m_{2}$ is the total mass of the transducer side, $l_{a}(t)$ is the active length between the pendulum's centre of mass and the pivot, $l_{a}(0)$ is the constant parameter of original $l_{a}$ at rest, $l_{b}$ is the active length between the transducer side's centre of mass and the pivot, and $g$ is the acceleration due to gravity. Under dynamic response, $l_{a}(t)$ is represented by equation 7 and unbalance is induced in the lever beam.

$$
\begin{array}{r}
l_{a}(t)=l_{a}(0)-\operatorname{sgn}(\theta(t)) \Delta l_{a}(t) \\
\text { where, } \Delta l_{a}(t)=l \cos (\phi(t))
\end{array}
$$

where, $\Delta l_{a}(t)$ is the change in active length $l_{a}(t)$ when pendulum is in motion and $\phi=0.5 \pi-\theta$. As the lever beam rocks about the pivot as a function of time, the transducer side mass (magnet) moves against the closely placed fixed coils with displacement $y(t)$. For $l_{b} \gg y(t)$, small arc angle can be assumed and $y(t)$ can be approximated as simple vertical displacement. The mechanical work done against the electrical damping of the transducer and the electrical power extractable from the system can be estimated by the dynamic forces about the lever beam. Therefore, the governing equation of the system sums up to the following.

$$
\left(\left(m_{1}-m\right) g+m g \cos (\theta(t))\right) \cdot l_{a}(t)=l_{b}\left(m_{2} g+F_{3}(t)\right)
$$

The $F_{3}(t)$ term here is assumed to be approximately equal to the mechanical force from the torque caused due to imbalance in the lever when $\theta$ is non-zero. This assumption is true for an ideal transducer where conservation of energy holds during mechanical-to-electrical power conversion, while taking into account the various damping terms.

For an electromagnetic transducer, displacement is related to electrical power output $P_{\text {elec }}$ by a squared relationship [16]; that is, $\theta^{2} \propto y^{2} \propto P_{\text {elec }}$. An estimate of the theoretical maximum electrical power output achievable $P_{\text {maxelec }}$, under ideal electrical load conditions (when electrical damping $D_{e}$ equals parasitic damping $D_{p}$ ) is assumed in equation 9 . For full derivation, refer to $[1,2,16]$. 


$$
P_{\text {maxelec }} \approx \frac{\left(m^{\prime} a^{\prime}\right)^{2}}{8 D_{p}}
$$

where, $m^{\prime}$ is a generic mass and $a^{\prime}$ is the time-varying-acceleration of this mass. The generic ' $m$ ' $a^{\prime}$ ' term from this equation is the mechanical force experienced by an ideal electromagnetic transducer. Therefore, $m^{\prime} a^{\prime} \approx F_{3}$ and an estimate of the maximum electrical power output can be calculated by substituting this term back into equation 8 to obtain the $\theta(t)$ dependent power output relationship in equation 10. $\theta(t)$ itself is determined by one of the equations 3 to 5 , depending on the excitation criteria.

$$
P_{\text {maxelec }}(t) \approx \frac{1}{8 D_{p}} \cdot\left(\frac{\left(\left(m_{1}-m\right) g+m g \cos (\theta(t))\right) \cdot l_{a}(t)}{l_{b}}-m_{2} g\right)^{2}
$$

The actual amount of maximum power extractable at the load $\left(P_{\text {maxload }}\right)$ also depends on the resistive loading conditions and the electrical damping $\left(D_{e}\right)$ of the electromagnetic transduction as defined in equations 11 and 12 respectively $[1,2]$. Where, $R_{\text {load }}$ is the resistive load, $R_{\text {coil }}$ is the resistance of the coil, $N$ number of coil turns, $l_{\text {coil }}$ is the length of the coil, $B$ is the flux density, $L_{\text {coil }}$ is the inductance of the coil. The imaginary component of equation 12 can be neglected for frequency $<1 \mathrm{kHz}$ for circular coils.

$$
\begin{gathered}
P_{\text {maxload }}=P_{\text {maxelec }} \cdot \frac{R_{\text {load }}}{R_{\text {load }}+R_{\text {coil }}} \\
D_{e}=\frac{\left(N l_{\text {coil }} B\right)^{2}}{R_{\text {load }}+R_{\text {coil }}+j \omega L_{\text {coil }}}
\end{gathered}
$$

While $D_{e}$ directly resists $y(t)$, it also has a fractional effect on $\theta(t)$ as it restricts the dynamic motion of the lever. The actual efficiency of the system and the transducer as well as additional nonlinear damping factors further reduce the maximum power estimated above. Therefore, various fitted numerical factors (either constants or functions of displacements) are required as coefficients for variables such as $F_{3}(t)$, $D_{p}, D_{e}$ and the feedback damping from $D_{e}$ to the pendulum damping in order for the numerical model to provide a more realistic estimate and to match with the experimental model.

\section{Numerical simulation}

A numerical model using MATLAB Simulink was constructed with numerical parameters in Table 2 to investigate the behaviour of the PEVEH design (in figure 3) under various excitation conditions.

Table 2: System parameters employed in the numerical simulation.

\begin{tabular}{|l|c||l|c|}
\hline$m(\mathrm{~kg})$ & 0.51 & $R_{\text {coil }}(\mathrm{k} \Omega)$ & 5.00 \\
\hline$m_{1}(\mathrm{~kg})$ & 0.61 & $R_{\text {load }}(\mathrm{k} \Omega)$ & 5.00 \\
\hline$m_{2}(\mathrm{~kg})$ & 0.31 & $c\left(\mathrm{Nsm}^{-1}\right)$ & 0.2 \\
\hline$l(\mathrm{~m})$ & 0.06 & $D_{p}\left(\mathrm{Nsm}^{-1}\right)$ & 3 \\
\hline$l_{a}(\mathrm{~m})$ & 0.100 & Feedback $D_{p}$ to $c$ & 1 \\
\hline$l_{b}(\mathrm{~m})$ & 0.200 & Power efficiency & 0.5 \\
\hline$f_{n}(\mathrm{~Hz})$ & 2.04 & \multicolumn{3}{|l}{} \\
\cline { 1 - 2 }
\end{tabular}

A qualitative comparison of angular displacement build up of the pendulum in time domain as a result of ordinary and parametric resonances near critical damping is presented in figures $5 \mathrm{a}$ and $5 \mathrm{~b}$ respectively. Parametric resonance, intrinsically, has a longer transient state. However, it can potentially accumulate to larger displacement amplitudes. As already established in the previous section, the output power response is directly proportional to displacement squared. Therefore, the effect of increasing oscillatory amplitude is amplified in the rise of power peak by this squared relationship. Figures 6a and $6 \mathrm{~b}$ qualitatively compares the power responses of the system for both cases in the frequency domain.

It can be observed that nonlinearity in parametric resonance plays a more significant role and is even seen at low amplitudes. On the other hand, the nonlinearity associated with ordinary resonance only becomes significant at high amplitudes. Therefore, for a given excitation amplitude, the parametric case exhibits a relatively wider operational frequency band. However, the higher nonlinear peaks on the left-hand-side of the natural frequency mark line in figure $6 \mathrm{~b}$ is only achievable either when an initial 


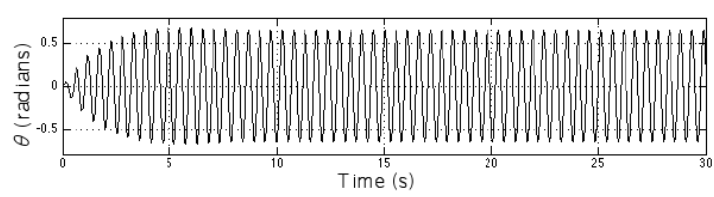

(a) Ordinary (numerical)

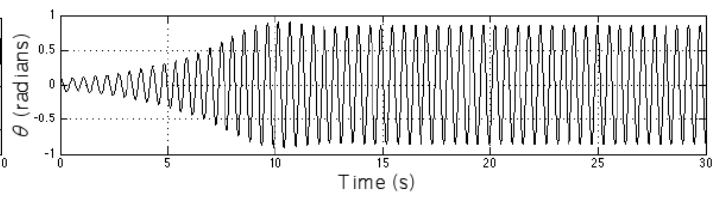

(b) Parametric (numerical)

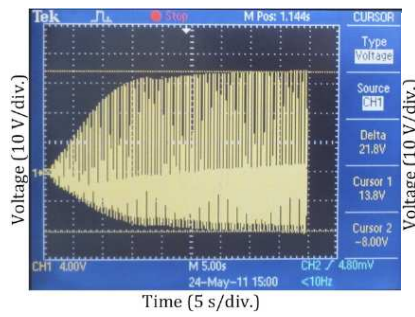

(c) Ordinary (experimental),(d) Parametric (experimen$\mathrm{V}_{p p}=21.8 \mathrm{~V} \quad$ tal), $\mathrm{V}_{p p}=56.4 \mathrm{~V}$

Figure 5: Numerical simulation and experimental results (induced with comparable excitation levels) of the oscillatory amplitude build up (in time domain) for the prototype near critical damping. Parametric resonance has a longer transient state but is able to attain a higher amplitude.

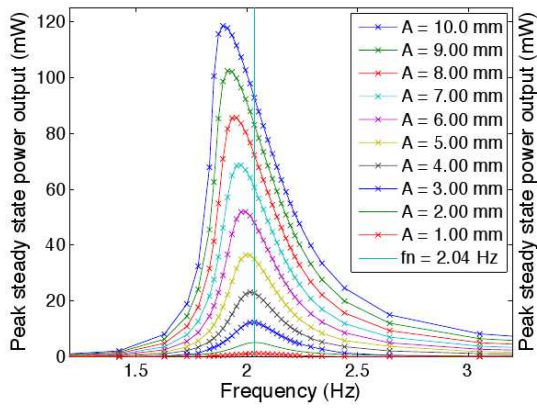

(a) Ordinary resonance

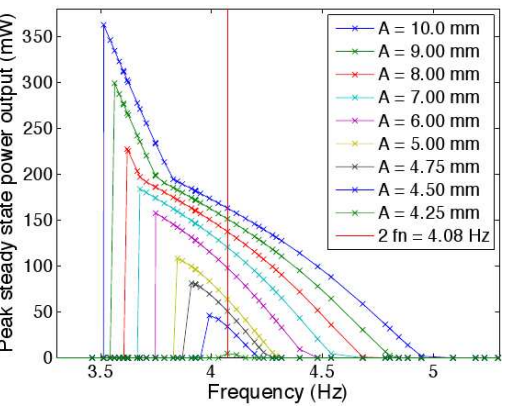

(b) Parametric resonance

Figure 6: Comparison between the numerically computed response for parametric resonance and ordinary resonance in the frequency domain. Onset of nonlinearity, and therefore the widening of frequency band, around natural frequency $f_{n}$ is relatively gradual for ordinary resonance with increasing excitation amplitude $A$. On the other hand, parametric resonance demonstrates relatively more significant nonlinearity even at low $A$ followed with the onset of higher orders of nonlinearity (steeper peaks) at higher $A$. However, the latter has zero steady state response immediately outside the frequency band and/or when $A$ is below an initiation threshold amplitude, which is around $4.25 \mathrm{~mm}$ in this setting.

displacement is present or during a downward frequency sweep. This is because during an upward frequency sweep, initial system displacement is absent upon reaching these otherwise operational frequency band; in other words, the system is trapped at a lower bifurcation point.

A steep jump (the elongated peak shape) in the nonlinear peak is observed at high excitation amplitudes in figure $6 \mathrm{~b}$, suggesting the onset of higher orders of nonlinearity. A theoretical explanation for this behaviour is that at these large amplitudes, pendulum oscillations no longer approximate to simple harmonic motion but undergo Hopf bifurcation to a limit cycle motion [10], hence, yielding an even faster growth in peak power levels.

With increase in excitation amplitude, the oscillatory amplitude (hence the peak power) also increases accordingly. For ordinary resonance, a second order polynomial relationship is present between displacement amplitude and power growth due to the $\theta^{2} \propto P$ relationship. However, the displacement amplitude growth is exaggerated with a higher order nonlinear factor for parametric resonance as demonstrated in the quantitative comparison in figure 7. Furthermore, an additional steep jump in amplitude growth rate for parametric resonance at high excitation amplitudes can be observed. This suggests the onset of further higher orders of nonlinearity and is in agreement with the observation in the figure $6 \mathrm{~b}$.

Evidently, the numerical simulations have demonstrated that parametric resonance has modestly 


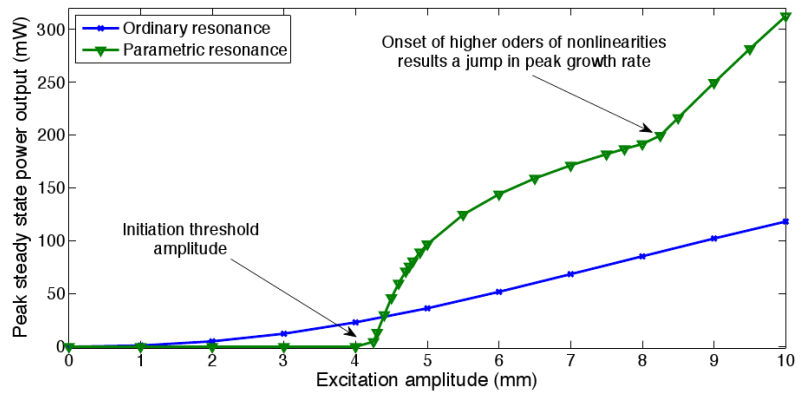

Figure 7: Quantitative numerical comparison between the peak power response for ordinary and parametric resonance to varying excitation amplitudes. Beyond a certain threshold of the excitation amplitude, parametric resonance rapidly outperforms ordinary resonance.

broader operational frequency band as a result of more significant nonlinearities and higher achievable power peaks than its ordinary resonance counterpart. However, it should be noted that 'an order higher' in performance as described in Section II does not necessarily denote absolute power magnitudes but more essentially the higher order polynomial behaviour demonstrated in figure 7 . In fact, when the excitation amplitude just marginally exceeds the required initiation threshold amplitude, the absolute peak power achievable is lower than its ordinary counterpart. Therefore, the parametric approach is increasingly rewarding at higher excitation amplitudes.

\section{Experimental prototype}

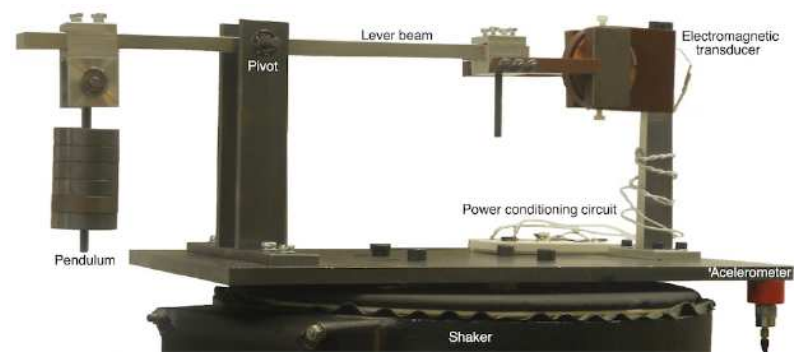

Figure 8: Preliminary PEVEH prototype.

To verify the theoretical and numerical predictions, an initial macro scale electromagnetic prototype (shown in figure 8) with system parameters listed in Table 3 was constructed and studied. The unmeasured parameters in Table 3 were numerically estimated and fitted in order to match the numerical model with the experimental power response.

Table 3: System parameters of the experimental prototype and fitted values of the corresponding numerical model (to match the recorded power response).

\begin{tabular}{|l|c||l|c|}
\hline \multicolumn{2}{|c||}{ Measured } & \multicolumn{2}{c|}{ Numerically fitted } \\
\hline$m(\mathrm{~kg})$ & 0.71 & $c\left(\mathrm{Nsm}^{-1}\right)$ & 0.2 \\
\hline$m_{1}(\mathrm{~kg})$ & 1.0 & $D_{p}\left(\mathrm{Nsm}^{-1}\right)$ & 5.4 \\
\hline$m_{2}(\mathrm{~kg})$ & 0.41 & $D_{e}\left(\mathrm{Nsm}^{-1}\right)$ & 100 \\
\hline$l(\mathrm{~m})$ & 0.07 & $D_{e}$ coefficient & $0.06(|\theta|+1)^{2}$ \\
\hline$l_{a}(\mathrm{~m})$ & 0.102 & Feedback $D_{p}$ to $c$ & 0.15 \\
\hline$l_{b}(\mathrm{~m})$ & 0.255 & Peak power efficiency (parametric) & 0.45 \\
\hline$f_{n}(\mathrm{~Hz})$ & 1.88 & Peak power efficiency (ordinary) & 0.15 \\
\hline$R_{\text {coil }}(\mathrm{k} \Omega)$ & 5.20 & \multicolumn{2}{|}{} \\
\hline$R_{\text {load }}(\mathrm{k} \Omega)$ & 5.40 & & \\
\hline
\end{tabular}

The transducer has a total component volume of around $50 \mathrm{~cm}^{3}$ and practical device volume of nearly $90 \mathrm{~cm}^{3}$. A four-magnet arrangement [17] was employed. The magnets are disc-shaped sintered 
Neodymium Iron Boron with dimensions of $22 \mathrm{~mm}$ diameter and $10 \mathrm{~mm}$ depth. The coil is also cylindrical in shape with dimensions of $50 \mathrm{~mm}$ outer diameter, $5 \mathrm{~mm}$ inner diameter, $10 \mathrm{~mm}$ depth, 90 microns wire diameter and an estimated coil turns of approximately a quarter of a million. The prototype's total component volume is approximately $500 \mathrm{~cm}^{3}$ and its practical device volume is around $1,800 \mathrm{~cm}^{3}$.

At ideal load resistance of $5.4 \mathrm{k} \Omega$, excitations in excess of $0.4 \mathrm{~ms}^{-2}$ brought about the onset of the principal order parametric resonance. The peak electric power recorded at parametric resonance is $956.6 \mathrm{~mW}$ at $1.70 \mathrm{~ms}^{-2}$ and at ordinary resonance is $27.75 \mathrm{~mW}$ at $0.65 \mathrm{~ms}^{-2}$. Furthermore, parametric resonance at this excitation setting (from which the peak power figure was noted) did not reach a steady state but was rather constrained by the physical limits of the design, which only permitted the pendulum to exhibit a maximum angular displacement of $\pm \frac{\pi}{2}$ radians. If larger angular displacements or circular motion are accommodated, then even higher power levels can be achieved.

The qualitative comparison of experimental oscillatory amplitude build up shown in figures $5 \mathrm{c}$ and $5 \mathrm{~d}$ is in agreement with their numerical counterparts with regard to a longer transient state for the parametric case. However, the eventual steady state accumulated to a much higher power level than ordinary resonance. Also, at higher excitation, the time required to attain peak amplitude is shorter.

The experimental Bode plots of power responses are shown in figure 9. At similar excitation levels (see Table 4), parametric resonance yielded over 6 times higher peak power than ordinary resonance. The mechanical shaker employed had a physical limit of approximately $5 \mathrm{~mm}$ in amplitude. Within this constraint, ordinary resonance failed to demonstrate observable nonlinearities. The operational frequency bandwidth is measured from half power points. Figure 10 contrasts the frequency bandwidth and extractable power for both resonances at similar input acceleration levels $\left(\sim 0.6 \mathrm{~ms}^{-2}\right)$. In this scenario, the parametrically driven system exhibited around $50 \%$ increase in the operational frequency band. Taking the ordinary resonance half power points as reference, the parametric case power curve experienced nearly a 3-fold broader frequency bandwidth.

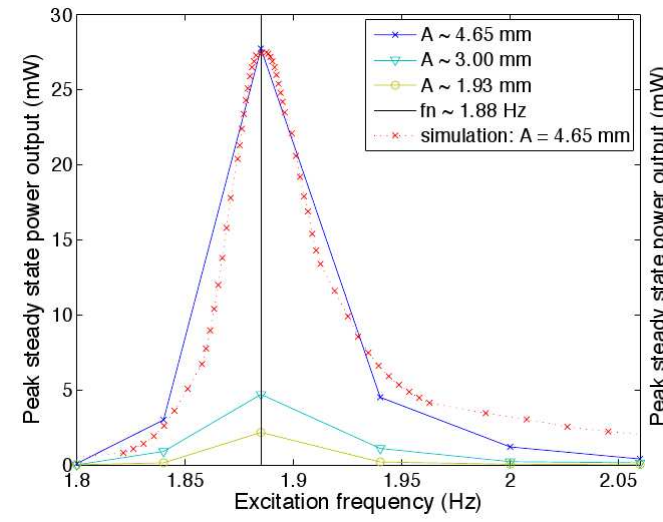

(a) Ordinary resonance

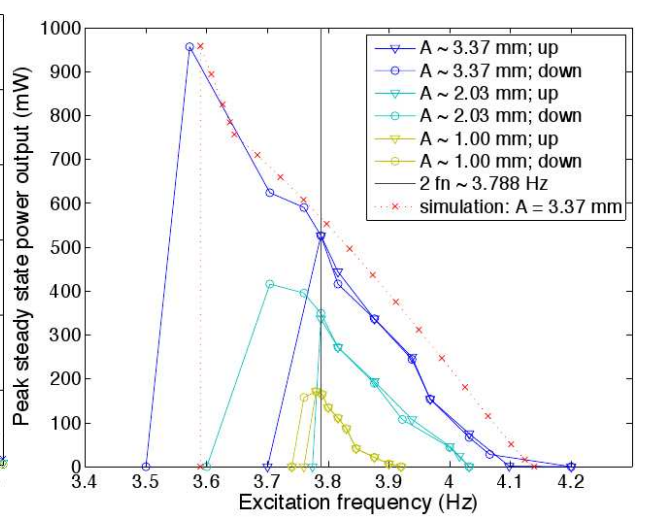

(b) Parametric resonance

Figure 9: Experimental power response in frequency domain for various excitation amplitudes $A$. The fitted simulation equivalent of the recorded peak power data are also plotted. With higher $A$, nonlinearity associated with parametric resonance rapidly becomes significant and results in the widening of frequency bandwidth, while that of ordinary resonance remains relatively confined. The steep jump of the nonlinear peak associated with parametric resonance at high amplitudes as described in figure $6 \mathrm{~b}$ is validated. The nonlinear peaks have different responses during upward and downward frequency sweeps because they are only achievable when significant initial displacements are present to allow the system to jump to a higher bifurcation point.

\section{Discussion}

The PEVEH prototype has experimentally performed an order better at parametric resonance than at ordinary resonance, confirming the theoretical and numerical predications of its advantages. A summarised comparison of the merits and demerits of the two cases are presented in Table 5. Apart from comparing with itself, Table 6 briefly contrasts the prototype's performance against selected current state-of-the-art macro-sized electromagnetic vibration energy harvesters. The preliminary experimental results reported here already compares favourably against the current state-of-the-art. Therefore, this serves as a motivation for further research in applying parametric resonance for vibration energy harvesting. 


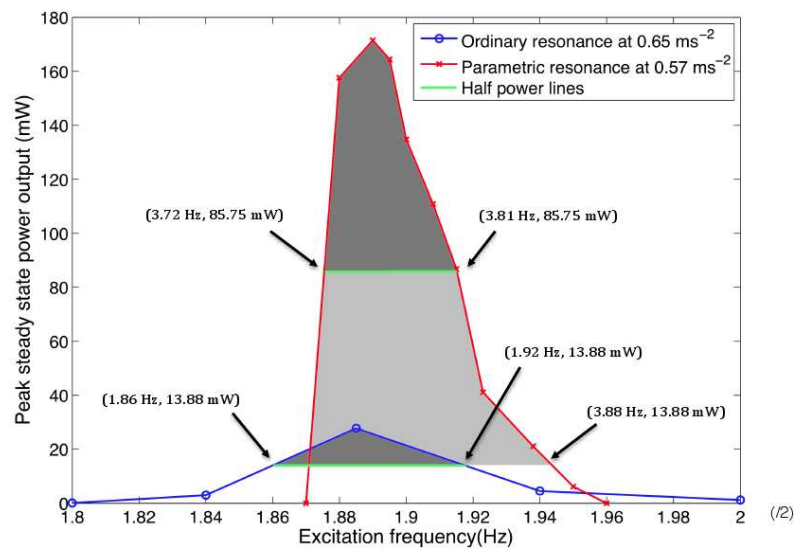

Figure 10: Experimental frequency bandwidth and extractable power of parametric resonance (frequency scale halved for the purpose of comparison) and ordinary resonance at comparable accelerations $\left(\sim 0.6 \mathrm{~ms}^{-2}\right)$. The darker shaded regions denote extractable power within the half power bands. In absolute terms, ordinary and parametric resonances have half power bands of 0.06 and $0.09 \mathrm{~Hz}$ ( $\sim 50 \%$ increase) respectively. The lighter shaded region represents the additional potential power extractable by parametric resonance above ordinary resonance's half power point (bandwidth $\sim 0.16 \mathrm{~Hz}$, nearly 3 times wider).

Table 4: Comparison of ordinary and parametric resonances' experimental performance. The latter has demonstrated over 6 times higher absolute peak power (at comparable acceleration $\sim 0.6 \mathrm{~ms}^{-2}$ ) and also performed an order better in terms of power density normalised against acceleration squared. Higher accelerations for ordinary resonance were not measured because the shaker's physical amplitude limit of nearly $5 \mathrm{~mm}$.

\begin{tabular}{c|ccccc}
\hline \multirow{5}{*}{ Ordinary } & $\begin{array}{c}\text { Peak power } \\
(\mathrm{mW})\end{array}$ & $\begin{array}{c}\text { Frequency } \\
(\mathrm{Hz})\end{array}$ & $\begin{array}{c}\text { Amplitude } \\
(\mathrm{mm})\end{array}$ & $\begin{array}{c}\text { Acceleration } \\
\left(\mathrm{ms}^{-2}\right)\end{array}$ & $\begin{array}{c}\text { Normalised Power Density } \\
\left(\mu \mathrm{Wcm}^{-3} \mathrm{~m}^{-2} \mathrm{~s}^{4}\right)\end{array}$ \\
\hline \multirow{3}{*}{ Parametric } & 2.17 & 1.88 & 1.93 & 0.27 & $1.65 \mathrm{E}+01$ \\
& 4.70 & 1.88 & 3.00 & 0.42 & $1.48 \mathrm{E}+01$ \\
& 27.75 & 1.88 & 4.65 & 0.65 & $3.65 \mathrm{E}+01$ \\
\hline & 171.5 & 3.78 & 1.00 & 0.57 & $2.93 \mathrm{E}+02$ \\
& 415.9 & 3.704 & 2.03 & 1.1 & $1.91 \mathrm{E}+02$ \\
\hline
\end{tabular}

As mentioned in Section I, Daqaq et al. (2009) [5] appears to be the first and only literature to date that has investigated the employment of parametric excitation for vibration energy harvesting. Despite providing a thorough and crucial theoretical analysis, a groundbreaking leap forward in practical performance has yet to be reported. The main limitation of a parametrically excited system is the need for the excitation amplitude to overcome an initial threshold; below which, steady state response will be zero. Daqaq et al. [5] has provided a comprehensive analytical model for this threshold amplitude.

The initiation threshold amplitude issue is not unique to Daqaq et al.'s parametrically excited cantilever and is intrinsic to most parametrically excited systems. However, the two-degrees-of-freedom PEVEH design reported here is less constrained by this shortcoming. This is because the principal damping in the system acts as the key contributor to this limitation (and the threshold is nonexistent for a theoretically undamped scenario). For PEVEH, the principal source of damping (transducer) acts on the secondary oscillating element (lever beam). So the excitation of the primary oscillating element (pendulum) is on a different degree-of-freedom and the effect of initial damping is minimised. In turn, this implies that a relatively higher initiation threshold amplitude is required if the principal source of damping is on the same degree-of-freedom as is the case for Daqaq et al..

The requirement of a non-zero initial displacement (to 'push' the system out of stable equilibrium) is another intrinsic property of most parametrically excited systems. A design that places the rest position in an unstable equilibrium could serve as a solution.

Parametrically driven harvesters, despite their potential capabilities of exhibiting significantly higher performance, are not perfect. Therefore, the integration of both direct and parametric excitations to compensate and complement each other, can serve as an ideal solution for vibration energy harvesting. 
Table 5: A summarised comparison between ordinary and parametric resonances.

\begin{tabular}{l|cc}
\hline & Ordinary & Parametric \\
\hline Peak power density normalised against acceleration & lower & an order higher \\
\hline $\begin{array}{l}\text { Increase in nonlinearity and frequency bandwidth } \\
\text { with amplitude growth }\end{array}$ & not observed & immediately observable \\
\hline Transient state & shorter & prolonged \\
\hline Initiation threshold amplitude requirement & no & yes \\
\hline Non-zero initial displacement requirement & no & yes \\
\hline
\end{tabular}

Table 6: Comparing PEVEH with selected current state-of-the-art macro-sized electromagnetic vibration energy harvesters in terms of power density normalised against acceleration squared.

\begin{tabular}{|c|c|c|c|c|c|}
\hline Reference & $\begin{array}{l}\text { Peak power } \\
(\mathrm{mW})\end{array}$ & $\begin{array}{l}\text { Freq. } \\
(\mathrm{Hz})\end{array}$ & $\begin{array}{l}\text { Volume } \\
\left(\mathrm{cm}^{3}\right)\end{array}$ & $\begin{array}{l}\text { Acceleration } \\
\left(\mathrm{ms}^{-2}\right)\end{array}$ & $\begin{array}{l}\text { Normalised Power Density } \\
\qquad\left(\mu \mathrm{Wcm}{ }^{-3} \mathrm{~m}^{-2} \mathrm{~s}^{4}\right)\end{array}$ \\
\hline PEVEH (parametric) & 171.5 & 3.57 & 1,800 & 0.57 & $2.93 E+02$ \\
\hline Perpetuum (2008) [1, 18] & 1.000 & 100 & 135 & 0.25 & $1.19 \mathrm{E}+02$ \\
\hline Lumedyne (2008) $[1,19]$ & 1.000 & 53 & 27 & 1 & $3.70 \mathrm{E}+01$ \\
\hline PEVEH (ordinary) & 27.75 & 1.88 & 1,800 & 0.65 & $3.65 E+01$ \\
\hline Ferro Sol. (2009) [20] & 5.270 & 60 & 170 & 0.98 & $3.23 \mathrm{E}+01$ \\
\hline Hadas (2007) $[1,21]$ & 3.500 & 34.5 & 45 & 3.1 & $8.09 \mathrm{E}+00$ \\
\hline Waters (2008) [22] & 18.00 & 90 & 27 & 9.81 & $6.93 \mathrm{E}+00$ \\
\hline Glynne-Jones (2001) [1, 23] & 2.800 & 106 & 3.66 & 13 & $4.53 \mathrm{E}+00$ \\
\hline
\end{tabular}

\section{Future work}

Ongoing and future work involves miniaturising the macro-sized prototype as well as exploring thickfilm and MEMS implementations of parametrically excited vibration energy harvesters. Future research could revolve around scaling effects and the effectiveness of applying parametrically excited harvesters to real world infrastructural vibration.

Furthermore, the phenomenon of autoparametric resonance is also being explored. The presence of a directly excited component within such working mechanisms reduces the initiation threshold amplitude and helps to overcome the requirement of a non-zero initial displacement. Therefore, this can complement a parametrically excied harvester's shortcomings while exploiting its potential performance advantages.

\section{Conclusion}

This paper has investigated the feasibility of employing parametric resonance for vibration energy harvesting. The numerical simulations and experimental prototype constructed have verified the theoretical predication of an order higher in oscillatory amplitude (hence power) growth than ordinary resonance. Experimentally recorded peak power at parametric resonance $\left(171.5 \mathrm{~mW}\right.$ at $\left.0.57 \mathrm{~ms}^{-2}\right)$ have outperformed ordinary resonance $\left(27.75 \mathrm{~mW}\right.$ at $\left.0.65 \mathrm{~ms}^{-2}\right)$ by an order of magnitude in terms of power density normalised to the squared input acceleration. The growth of significant nonlinearities with increasing amplitude also demonstrated $50 \%$ increase in operational frequency bandwidth measured from their respective half power points (or nearly 3 folds, taking the half power point for the ordinary response as the reference). Additionally, these initial experimental results already compare favourably with respect to the current state-of-the-art.

\section{Acknowledgements}

This work was supported by the Engineering and Physical Sciences Research Council [grant number $\mathrm{EP} / \mathrm{I} 019308 / 1]$. 


\section{References}

[1] S. Priya and D. Inman, Energy Harvsting Technologies. New York: Springer US, 2009. [Online]. Available: http://dx.doi.org/10.1007/978-0-387-76464-1_5

[2] S. Beeby, M. Tudor, and N. White, "Energy harvesting vibration sources for microsystems applications," Meas. Sci. Technol., vol. 17, no. 12, pp. R175-R195, 2006.

[3] P. Mitcheson, E. Yeatman, G. Rao, A. Holmes, and T. Green, "Energy harvesting from human and machine motion for wireless electronic devices," Proc. IEEE, vol. 96, no. 9, pp. 1457-1486, 2008.

[4] G. Ye and K. Soga, "Energy harvesting from water distribution systems," J. Energy Eng., p. 30pp., 2011, in press.

[5] M. Daqaq, C. Stabler, Y. Qaroush, and T. Seuaciuc-Osorio, "Investigation of power harvesting via parametric excitations," J. Intel. Mat. Syst. Str., vol. 20, no. 5, pp. 545-557, 2009.

[6] M. Faraday, "On a peculiar class of acoustical figures and on certain forms assumed by a group of particles upon vibrating elastic surfaces," Phil. Trans., pp. 299-318, 1831.

[7] N. Minorsky, Nonlinear Oscillations. New York: Robert E. Krieger, 1974.

[8] L. Rayleigh, "On maintained vibration," Philos. Mag., vol. 15, no. 94, pp. 229-235, April 1883.

[9] A. Nayfeh and D. Mook, Nonlinear Oscillations. New York: Wiley-Interscience, 1979.

[10] A. Tondl, T. Ruijgrok, F. Verhulst, and R. Nabergoj, Autoparametric resonance in mechancial systems. Cambridge, UK: Cambridge University Press, 2000.

[11] N. Minorsky, Introduction to Non-linear Mechanics. Michigan: Edwards Brothers, 1947.

[12] E. Butikov, "Parametric resonance in a linear oscillator at square-wave modulation," Eur. J. Phys., vol. 26 , no. 1, pp. 157-174, 2005.

[13] L. Oropeza-Ramos and K. Turner, "Parametric resonance amplification in a memgryoscope," in Proceedings of IEEE Sens., Irvine, CA, 30 October - 3 November 2005, p. 4pp.

[14] M. Sharma, E. Sarraf, and E. Cretu, "Parametric amplification/damping in mems gyroscopes," in Proceedings of the 24th IEEE Int. Conf. MEMS, Cancun, 17 March 2011, pp. 617-620.

[15] V. Milkovic. (2005) Two stage mechanical amplifier: pendulum lever system. [Online]. Available: http://www.veljkomilkovic.com/OscilacijeEng.html

[16] C. Williams and R. Yates, "Analysis of a micro-electric generator for microsystems," Sens. Actuators $A$, vol. 52, no. 1-3, pp. 8-11, 1996.

[17] S. Beeby, R. Torah, M. Tudor, P. Glynne-Jones, T. O'Donnell, C. Saha, and S. Roy, "A micro electromagnetic generator for vibration energy harvesting," J. Micromech. Microeng, vol. 17, no. 7, pp. 1257-1265, 2007.

[18] Perpetuum - products, pmg-17. [Online]. Available: http://www.perpetuum.com/products.asp

[19] Lumedyne technolgies - energy harvester. [Online]. Available: http://www.lumedynetechnologies. com/EnergyHarvester.html

[20] Ferro solutions veh-460. [Online]. Available: http://www.ferrosi.com/files/VEH460_May09.pdf

[21] Z. Hadas, M. Kluge, V. Singule, and C. Ondrusek, "Electromagnetic vibration power generator," in Proceedings of IEEE Symp. Diagn. Electr. Mach. Power Electron. Drives 2007, Cracow, 6-8 September 2007, pp. 451-455.

[22] R. Waters, B. Chisum, H. Jazo, and M. Fralick, "Development of an electro-magnetic transducer for energy harvesting of kinetic energy and its' applicability to a mems-scale device," in Proceedings of Nanopower Forum 2008, Costa Mesa, California, 02-04 June 2008, pp. 359-374.

[23] P. Glynne-Jones, "Vibration powered generators for self-powered microsystems," Ph.D. dissertation, University of Southampton, 2001. 